te ritiene il liquido: e ciô si vede manifestamente traguardando un perxo di carta di riso seccala a intervalli. Nei disegni fatti ad acquerello sulla carta di cui parliamo si sà che le tinte sono delicatissime e li effetti molto vellutati in guisa che questa sostanza è statz ricercala soprattutto dalle Signore, che amano per lo più questa specie di ombreggiatura all' acquatinta.

In sul primo si penserebbe che $\mathrm{l}$ axione del pennello dovesse riempire le cellule di materia colorante, o che almeno si dovessero presentar colorate le membrane delle cellule stesse, però la cosa procede del tutto diversamente. Il colore si concentra negli ínterstizì delle cellule, nè giammai sî manifesta nell' interno di queste; è adunque da questa divisione microscopica del colore condensato nelle connessioni delle cellule, e delle aree incolore delle medesime che resulta il vellutato dei disegni. Per una tal colorazione ne segue che sopra un millimetro quadrato vi sono 20 piccole asperiti bianche, separate da aleretante periferie poligone colorate. La colorazione diviene quindi quasi un lavoro di mosaico, come evidentemente dimostra lt osservazione microscepica.

Dotc. Arthe Tasst.

\title{
AVORTO VEGETABILE
}

11 Prof. Carlo Morren ha comunicato all Acrademia delle Scienze di Bruselles un fatto assai curioso e che può aprire il campo ad un nuovo ramo d' industria. Le Palme del genere Phytelephas o Elephantasia originarie del Perù, producono dei grossí semi che dal volume d' una nocciola si estendono talvolta fno a quello della testa d'un bambino. L'albume che sul principio ha l'apparenza lattea si beve o si converte in ace10; dipoi indurisce alquanto e pù̀ mangiarsi come le mandorle; infine si ossifica e diviene un Avorio vegetabile eccellente. Il Prof. Morren ha studiata questa sosianza che può da noi ottenersi coltivando la pianta in stufa, e ne ha fatti fare diver- 
129

si oggetti come palle da biliardo, bottoni da camicia, tavolette da miniare ec. Quest' Avorio è più bianco di quello animale, senza grana, nè venature, è lucido e bellissimo. L'acqua lo $\mathbf{f a}$ aumentare di volume quasi del doppio, e lo rende trasparente, ma nell' asciugarsi ritorna duro; cosi i colori della miniatura penetrano veramente nella sua sostanza. La struttura poi di quest'avorio esaminata col microscopio, è una delle più curiose che si trovino nel regno vegetabile. Essa risulta di cellule in forma di stelle con 5 a 10 raggi ; le estremita dei raggi di una cellula combinano con le estremita di quelli di un'altra ec. lasciando cosi tanti spazj vuoti fra loro.

Articolo Communicato

dal Marchese Prof. Cosimo Ridorfi.

\section{ANALISI DEL TIH DEL SIG. PELIGO'T}

Le ricerche fatte sin qui sulla composizione del The, hanno provato che conteneva il tannino, un olio essenziale aromatico, e la teina, che si è riconosciuta identica colla caffeina che si trova nel caffe. $\mathbf{E}$ curioso che il The ed il caffe che ci servono oggi con tanta profusione per far due bevande, che secondo i diversi paesi si rimpiazzano reriprocamente, contengono una stessa sostanza. Il Sig. Peligot ha cominciato dal determinare le quantità $\mathrm{d}$ aznto che $\mathrm{i}$ diversi Thè contengono: questa quantità và da 6,58 a 5,10 per cento di Thè, ciò che porta a 20 o 30 per cento la proporzione di materia azotata che si sia sin ad ora trovata ne' vegetabili.

Secondo Peligot questa materia azotata sarebbe quasi unicamente la teina.

Oltre la teina, Peligot trova nel Thè l'acido pettico, un acido grasso e una sostanza analoga alla caseina che sarebbe unita al tannino. 